\title{
A Comparative Effectiveness Trial to Reduce Burnout and Improve Quality of Care
}

\author{
Michelle P. Salyers, Ph.D.*
}

Professor, Department of Psychology, Indiana University-Purdue University Indianapolis

(IUPUI); Director, ACT Center of Indiana

Jennifer M. Garabrant, B.S.W.

Program Manager, Department of Psychology and ACT Center of Indiana, IUPUI

Lauren Luther, M.S.

Clinical Psychology Graduate Student, Department of Psychology and ACT Center of Indiana, IUPUI

Nancy Henry, B.A.

Project Manager, Department of Psychology and ACT Center of Indiana, IUPUI

Sadaaki Fukui, Ph.D.

Associate Professor, Department of Social Work, School of Social Work and Center for Social Health and Well-being and ACT Center of Indiana, IUPUI

\section{Dawn Shimp}

Research Assistant, Department of Psychology and ACT Center of Indiana, IUPUI

Wei Wu, Ph.D.

Associate Professor, Department of Psychology and ACT Center of Indiana, IUPUI

Tim Gearhart, M.S.W.

Vice President of Clinic Operations, Pulaski Memorial Hospital

Gary Morse, Ph.D.

Vice President of Research and Development, Places for People, Inc.; Adjunct Professor, Department of Psychology, University of Missouri-St. Louis

Mary M. York, M.S.W.

Doctoral Student, Department of Psychology at Southern Illinois University-Carbondale

Angela L. Rollins, Ph.D.

Associate Research Professor, Department of Psychology and ACT Center of Indiana, IUPUI; Associate Director, Center for Health Information and Communication, Richard L. Roudebush VAMC; Investigator, Regenstrief Institute, Inc.

*Corresponding Author: Michelle Salyers; IUPUI School of Science, Department of Psychology, LD 124, 402 N. Blackford St., Indianapolis, IN, 46202; Phone: (317) 274-2904; Fax: (317) 2746756; email: mpsalyer@iupui.edu 
COMPLIANCE WITH ETHICAL STANDARDS:

FUNDING: Research reported in this publication was funded through a Patient-Centered Outcomes Research Institute (PCORI) Award (\# IH-1304-6597)

DISCLAIMER: The statements in this publication are solely the responsibility of the authors and do not necessarily represent the views of the Patient-Centered Outcomes Research Institute (PCORI), its Board of Governors or Methodology Committee.

CONFLICT OF INTEREST: The authors declare that they have no conflicts of interest.

ETHICAL APPROVAL: All procedures performed in studies involving human participants were in accordance with the ethical standards of the institutional and/or national research committee and with the 1964 Helsinki declaration and its later amendments or comparable ethical standards.

INFORMED CONSENT: Informed consent was obtained from all individual participants included in the study.

DATE OF REVISED SUBMISSION: 10/17/18 


\begin{abstract}
Clinician burnout is presumed to negatively impact healthcare quality; yet scant research has rigorously addressed this hypothesis. Using a mixed-methods, randomized, comparative effectiveness design, we tested two competing approaches to improve care - one addressing clinician burnout and the other addressing how clinicians interact with consumers - with 192 clinicians and 469 consumers at two community mental health centers. Although qualitative reports were promising, we found no comparative effectiveness for either intervention on burnout, patient-centered processes, or other outcomes. Discussion includes identifying ways to strengthen approaches to clinician burnout.
\end{abstract}

Keywords: burnout, randomized comparative effectiveness, quality of care, mental health 


\section{Introduction}

Professional burnout is commonly defined by Maslach's conceptualization of high levels of emotional exhaustion and depersonalization (negative or cynical attitudes about care recipients $^{1}$ ) and a diminished sense of personal achievement (1993). Burnout is common among clinicians, with rates of up to $67 \%$ of those working in mental health reporting high levels of burnout on the Maslach Burnout Inventory (Oddie, 2007; Rohland, 2000; Siebert, 2005; Webster \& Hackett, 1999). Research also indicates that burnout is associated with a number of problems not only for mental health clinicians (e.g., insomnia, relationship problems, poor mental health) (Carson et al., 1999; Heaney, Price, \& Rafferty, 1995; Killian, 2008; Long, Blackwell, \& Midgley, 1990; Perrone, Ægisdóttir, Webb, \& Blalock, 2006; Rohland, 2000; Smoot \& Gonzales, 1995; Stalker \& Harvey, 2002), but also for employer organizations (e.g., turnover), consumers (e.g., less empathic treatment providers), and the mental health system as a whole, resulting in poor quality of care. Thus, interventions that address burnout have the potential to positively impact quality of care and consumer outcomes; yet, little controlled research has directly addressed this hypothesis.

Guided by the self-determination theory of human motivation (Ryan \& Deci, 2000) and the Job Demands-Resources model of burnout (Bakker \& Demerouti, 2007; Bakker, Demerouti, de Boer, \& Schaufeli, 2003), we hypothesized that clinician burnout might affect patientcentered care, engagement, and consumer outcomes. Self-determination theory asserts that people are at their best when needs for autonomy, competence, and relatedness are met (Ryan \& Deci, 2000). In healthcare, for example, Williams and colleagues (2004) found that patients' perceived autonomy support from clinicians predicted patients' level of perceived competence in

\footnotetext{
${ }^{1}$ We refer to care recipients as "consumers" in mental health settings, and "patients" when referring to recipients of general medical care or when using specific terms of patient-centered care or patient activation.
} 
managing diabetes three months later. Competence predicted self-management behaviors and change in glucose level as well. Similar support has been found for smokers, where perceived autonomy support from clinicians predicted perceived competence, which was further associated with smoking cessation.(G. C. Williams et al., 2006) Patients should be most motivated and engaged in treatment when they feel supported to make choices, feel connected to their treatment provider, and feel competent to work towards better health. These patient-centered care processes may be jeopardized by clinician burnout.

According to the Job Demands-Resources model of burnout and conservation of resources (Bakker \& Demerouti, 2007; Hobfoll, 1989), job demands (e.g., interacting with consumers with intensive needs, balancing competing priorities) require effort over time and can have detrimental effects on the clinician (e.g., emotional exhaustion). As clinicians become exhausted, they conserve resources and pull back (leading to depersonalization). This conservation of resources can also lead to spending less time with consumers, and potentially being more directive and less supportive of consumers' autonomy. Depersonalization (cynical attitudes, treating consumers like objects) also may lead to lower expectations of consumers' abilities (seeing them as less competent) and make clinicians less able to form relationships with consumers (less relatedness). The lack of these therapeutic processes (low support for autonomy, relatedness, and competence) may lead to reduced engagement and, ultimately, worse outcomes.

Empirically, burnout has been associated with cognitive impairments in employees, including decreased attention (Van der Linden, Keijsers, Eling, \& Van Schaijk, 2005), which can lead to errors and less engagement with patients. Burnout has also been associated with decreased empathy (Åström, Nilsson, Norberg, Sandman, \& Winblad, 1991), a particularly salient component of many mental health interventions. For example, in a study of medical 
residents, reduced empathy mediated the relationship between burnout and lower self-ratings of patient-centered care (Passalacqua \& Segrin, 2012). Moreover, a recent meta-analysis found consistently negative relationships between burnout and quality of care $(\mathrm{r}=-0.26,95 \% \mathrm{CI}$ $[-0.29,-0.23])$ as well as safety $(\mathrm{r}=-0.23,95 \% \mathrm{CI}[-0.28,-0.17])($ Salyers et al., 2017). Although less research has directly assessed the link between clinician burnout and clinical outcomes, some studies have found detrimental effects of burnout. Halbesleben and Rathert (2008) surveyed 178 matched pairs of physicians, with patients who had recently been hospitalized; path analyses showed that the depersonalization component of physician burnout was related to lower patient satisfaction and longer patient reported recovery times. In the mental health field, Priebe and colleagues (2004) studied 24 assertive outreach teams working with people with severe mental illness and found that team level burnout was associated with increased hospitalization of consumers 9 months later.

There are solid theoretical reasons and growing empirical evidence (e.g., (Halbesleben \& Rathert, 2008; Passalacqua \& Segrin, 2012; Priebe et al., 2004; Salyers et al., 2017) to suggest that by targeting mental health clinician burnout we can improve patient-centered processes, engagement in care, and consumer outcomes. Yet, despite the high prevalence of burnout, relatively few interventions or prevention programs have been implemented, and very few have been adequately evaluated. Prior to the current study, a literature review (Morse, Salyers, Rollins, Monroe-DeVita, \& Pfahler, 2012) found 8 studies focusing specifically on reducing burnout in mental health clinicians, only two of which involved a randomized controlled trial (RCT); further, only two were conducted in the US and only one (Salyers et al., 2011) focused on community mental health clinicians. Five of the 8 studies (including one RCT) found significant reductions in burnout. The type of intervention varied, including communication 
training, empathy building, worksite social support development, and managerial support. The small number of studies and their methodological limitations make generalization difficult. In a more recent meta-analysis, Dreison and others (2018) identified 27 studies that had assessed the impact of an intervention on burnout in mental health settings; interventions, particularly those directed at the individual clinician, had positive, but small, effects on clinician burnout (overall, Hedges' $g=.13, p=.006)$. In addition, studies were limited in rigor of design, length of followup, and breadth of outcomes assessed.

Drawing from the literature and clinical experience, the Burnout Reduction: Enhanced Awareness, Tools, Handouts, and Education (BREATHE) program was designed to reduce community mental health clinician burnout (Salyers et al., 2011). This program integrates a combination of strategies designed to enhance personal resources to deal with stressful work situations, including traditional cognitive behavioral techniques, mindfulness practices, social skills training, and other self-care strategies in a workshop setting. A pilot test of BREATHE (Salyers et al., 2011) showed significant reductions in emotional exhaustion and depersonalization (cynicism) along with significant increases in optimistic attitudes regarding consumers. A second study tested BREATHE in a more rigorous design, randomizing participants to either BREATHE or a person-centered planning intervention (Rollins et al., 2016). Although there were no significant differences between groups following the intervention, BREATHE participants (unlike the person-centered planning group) did experience significant improvements in depersonalization at six weeks and in emotional exhaustion and positive expectations for consumers at 6 months. However, both of those prior studies only assessed clinician outcomes; patient-centered processes of care or consumer outcomes were not examined in relation to burnout or in response to the BREATHE intervention. 
The purpose of the current study was to test whether BREATHE could not only improve clinician outcomes, but also processes of care and consumer outcomes as well. Essentially we sought to test an alternative paradigm for healthcare administration, practice, and research, as suggested elsewhere (Bodenheimer \& Sinsky, 2014): that providing effective interventions for the health and well-being of clinicians is another way to systematically improve the quality of care they provide and, ultimately, consumer outcomes. In light of policy emphasizing comparative effectiveness research (Wilensky, 2006) that directly compares two or more alternatives to improve care, we partnered with consumers and clinicians to test BREATHE using a randomized, comparative effectiveness design. BREATHE works by targeting burnout directly. We chose motivational interviewing (MI) training as the comparator approach, which should improve patient-centered care by giving clinicians skills to work with consumers to support self-determination, but might not have a large impact on burnout. By choosing such a comparison, we maintained the spirit of comparative effectiveness research in ensuring consumers in a real-world setting would have a good chance of experiencing improved outcomes in either condition (Sox \& Greenfield, 2009), while being able to test the theoretical impact of providing an intervention addressing clinician burnout at the same time. We hypothesized that BREATHE would be more effective than MI in reducing clinician burnout and that consumers who were served by clinicians in the BREATHE intervention would experience improved patient-centered processes (reporting more perceived autonomy support, working alliance), engagement (fewer appointments missed, stronger satisfaction), and outcomes (improved patient activation, depression/anxiety, and functioning).

\section{Methods}


Setting and Participants. The target populations included clinicians and consumers at two Midwestern community mental health centers. The rural location was a public, non-profit organization that employed approximately 230 staff at the time of the study, providing community-based substance abuse and mental health services to nearly 6000 consumers annually. The urban location was also a public, non-profit organization that employed approximately 260 staff serving nearly 4000 consumers annually. Both organizations provide case management, home-based and school-based services, supported employment, medication management, and outpatient individual and group services, including those following evidencebased guidelines. The BREATHE and MI trainings typically took place at a neutral location offsite to minimize distractions.

We first recruited clinicians (i.e., staff who provide direct care to consumers) and then recruited consumers linked with those clinicians. Flyers and brochures were distributed via agency email and posted in locations accessed by staff at the agencies, and research team members presented the study at team meetings. Clinicians who expressed interest met with a research team member to provide informed consent. Clinicians were randomized via random number generator to treatment group (receiving either BREATHE or MI), stratified by site. After completing the baseline survey, clinicians were informed via email which treatment condition they were assigned to and were provided two dates from which they could choose to attend the first training session; 192 completed the baseline measure and were included in analyses (See CONSORT flow diagram). A subset of clinicians who participated in the RCT and were still active employees also completed a qualitative interview after participation in the trial $(\mathrm{N}=21$ from BREATHE and $\mathrm{N}=21$ from $\mathrm{MI})$. A stratified sampling scheme was used to recruit a 
minimum of three participants from each condition $(n=2)$, from each recruitment wave $(n=3)$, from each site $(\mathrm{n}=2)$ for interviews.

We also recruited and enrolled adult consumer participants to assess changes in patientcentered care and outcomes in response to the clinician interventions. As clinicians agreed to participate, electronic medical records were used to create a list of consumers seen during the prior month. A subsample of consumers for each participating clinician who served adult consumers was randomly selected for recruitment (up to five). Consumers who were at least age 18 and did not decline were scheduled with a research team member to complete the informed consent process and initial interview. There were 3 consumers who were determined to be ineligible due to being too symptomatic to complete the interview and one who consented, but whose clinician did not participate; 469 consumers were included (See CONSORT flow diagram).

Clinicians completed online surveys at baseline, 3 months, 6 months, and 12 months to assess clinician and job characteristics, burnout, other measures of job-related well-being and perceived quality of care. The survey took approximately 30 minutes, and clinicians were given a $\$ 10$ gift card after each completed survey. We interviewed consumers at baseline, 6 months, and 12 months to assess patient-reported processes of care, engagement, and consumer outcomes (improved patient activation, depression/anxiety, and functioning). Consumers were given $\$ 20$ for completing each interview. Clinicians participating in the qualitative interviews were given a \$20 gift card. All procedures were approved by the Indiana University Institutional Review Board.

BREATHE Intervention. BREATHE uses a relapse prevention conceptual framework, and incorporates mindfulness, cognitive restructuring, boundary setting, and social support 
(Salyers et al., 2011). Basic information is presented in a group workshop setting, where a number of individual, dyadic, and group exercises are used to help participants to practice strategies. The training also provides participants with a relapse prevention conceptual framework for understanding burnout and basic prevention principles. Further, participants are provided with a Burnout Prevention and Work Wellness Toolkit, in which they are encouraged to identify personal burnout warning signs and triggers and to develop a personalized follow-up self-care plan to reduce burnout. Although the pilot BREATHE workshop was conducted in one day, this project split the training into 3 sessions for spaced exposure and practice of the materials, which may be important for sustaining changes (Awa, Plaumann, \& Walter, 2010). The initial workshop was 4 hours, followed by two 2-2.5-hour sessions spaced approximately one month apart. BREATHE was provided by two clinical psychologists and an advanced doctoral student in clinical psychology.

Motivational Interviewing (MI) Training. The comparator condition was MI training for clinicians, a therapeutic approach based on trans-theoretical models of change and Rogers' client-centered therapy (Miller \& Rollnick, 2002). Central to MI is a patient-centered clinical orientation (Allsop, 2007) that involves both a spirit of collaborative work with the consumer on goals of his or her choosing and the strategic use of certain communication techniques to help resolve ambivalence regarding positive behavior change or perhaps create ambivalence around troublesome behaviors when none exists. MI is grounded in the principles of providing accurate empathy, developing discrepancies between goals and behaviors, rolling with resistance, and avoiding argument. Training includes didactic and experiential activities (e.g., using value cards to clarify meaning and goals). In this study, we offered MI in the same format as BREATHE, with an initial workshop of 4 hours and two, 2-2.5 hour follow-ups. MI was provided by a 
clinical psychologist and a master's level social worker, both with prior experience training clinicians in MI.

Measures. We assessed background characteristics, including demographics, and jobrelated variables (e.g., job tenure, caseload size) and several outcome variables. The primary outcome for the comparative effectiveness trial was burnout. The widely-used Maslach Burnout Inventory (C. Maslach, Jackson, \& Leiter, 1996) measures three components of burnout: emotional exhaustion, depersonalization, and personal accomplishment. The subscales have shown good internal consistency, stability over time, and convergent validity with related constructs (C. Maslach et al., 1996). Burnout was considered to be the most proximal outcome impacted by the intervention and a potential pathway to other clinician and consumer outcomes.

Other job-related well-being measures included Job satisfaction assessed with 1 item from the Job Diagnostic Survey (Hackman \& Oldham, 1974). We asked about turnover intentions through two self-report items used in prior studies (Salyers et al., 2011; Salyers, Rollins, Kelly, Lysaker, \& Williams, 2013): "How often have you seriously considered leaving your job in the past six months?" (rated from $1=$ never to $6=$ several times a week) and "How likely are you to leave your job in the next six months?" (rated from 1=not likely at all to $4=$ =very likely). We assessed Work-Life Balance with a six-item measure adapted from an 18-item measure developed by Carlson et al. (2000). The measure assesses three types of conflict (time-, strain-, and behavior-based) and two directions (work conflict with family and family conflict with work) of balance. We used the Emotional Labor Scale (Diefendorff, Croyle, \& Gosserand, 2005), which includes 14 questions about the relationship between emotions and interactions with consumers. We created four questions to assess the importance and confidence in skills to mirror processes in MI. We created one set of questions for stress reduction, e.g., "How 
(important is it for you to / confident are you that you can) reduce your work-related stress right now?" and one set for working in a patient-centered way, i.e., "How (important is it for you to / confident are you that you can) consistently interact with consumers/clients in a relaxed, nonjudgmental way?" The Perceptions of Supervisory Support Scale gathered information on how supervision can be linked to burnout and consumer outcomes (Fukui, Rapp, Goscha, Marty, \& Ezell, 2014).

Perceived Quality of Care was assessed with a self-reported scale refined from a prior study (Salyers et al., 2015). For the current study, we added items from suggestions during preliminary focus groups about how stress affects interactions or outcomes for consumers (Luther et al., 2016). We also created a parallel version for consumers to complete. After factor analysis, both scales had 22 items; the consumer version had three distinct factors (PersonCentered Care, Negative Staff Interactions, and Inattentive Care), while the clinician scale had two (Person-Centered Care and Discordant Care). Both versions demonstrated adequate internal consistency and validity with measures related to satisfaction and the therapeutic relationship (Luther et al., in press).

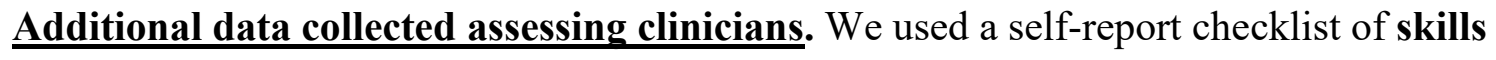
learned in the training tailored for the intervention received (BREATHE or MI) in each followup survey as a rudimentary fidelity check to help us assess the extent to which skills were being implemented from the clinicians' perspective. Agency Records were used to extract actual turnover at 6 and 12 months. Qualitative interviews (available from the corresponding author) were conducted with a subset of clinicians from each intervention arm (21 in each) to obtain feedback about the utility of each intervention and how the intervention may have impacted them 
and their work with consumers and co-workers. Interviews were conducted in person or by phone with a research assistant who was not involved in providing either intervention.

All consumer-reported measures were considered important secondary outcomes of the intervention and are listed below. We first describe measures assessing the processes of care, followed by consumer outcomes (symptoms, functioning, and hope).

Perceptions of patient-centered processes were assessed with two measures. For both, we prompted the consumer to report on the specific clinician from whose caseload they were randomly selected. We assessed perceived autonomy support with a 6-item version of the Health Care Climate Questionnaire (G. C. Williams, McGregor, King, Nelson, \& Glasgow, 2005). Consumers rated how much their clinicians support autonomy on a 7-point scale (strongly disagree to strongly agree). Perceived Relatedness was assessed with the short form of the consumer version of the widely used Working Alliance Inventory (WAI) (Tracey \& Kokotovic, 1989). The WAI has three subscales: task, bond, and goals.

Competence related to mental health management was assessed with the 13-item Patient Activation Measure-Mental Health (PAM-MH; (Hibbard, Mahoney, Stockard, \& Tusler, 2005) (e.g., "I know what each of my prescribed mental health medications does"). The PAM-MH has been shown to have strong test-retest reliability, correlates with related constructs (Green et al., 2010) and has been used in other samples of people with mental illness (Druss et al., 2010; Salyers, Matthias, et al., 2009).

Engagement was assessed with consumer satisfaction using the Client Satisfaction Questionnaire, an 8-item satisfaction checklist used in several large-scale community-based mental health studies, with good reliability (Bond et al., 1990). Engagement was also assessed by the proportion of scheduled appointments kept (using medical record information) and 
medication adherence (for consumers who are prescribed medications) was rated with the Medication Adherence Rating Scale (MARS), a 10-item scale with established reliability and validity in people with mental illness (Thompson, Kulkarni, \& Sergejew, 2000).

Perceived Quality of Care was assessed with subscales of the consumer version of the quality of care scale described above (Luther et al., in press).

Consumer outcomes. We selected brief measures of depression and anxiety as these are common concerns across a variety of consumers. The Patient Health Questionnaire 9-Item (PHQ-9) is a brief, widely used self-report assessment of depression developed for primary care (Kroenke, Spitzer, \& Williams, 2001; Spitzer, Williams, Kroenke, Hornyak, \& McMurray, 2000). Anxiety was assessed with the seven-item Generalized Anxiety Disorder (GAD-7) that has been validated in large primary care and population-based studies (Kroenke, Spitzer, Williams, Monahan, \& Lowe, 2007; Spitzer, Kroenke, Williams, \& Lowe, 2006). Physical and mental health functioning was assessed with the Short Form 12-Item Health Survey (SF-12) (Ware, Kosinski, \& Keller, 1996), a health-related quality of life measure that has been used extensively, particularly in depression management trials (Kroenke et al., 2010; J. W. Williams, Jr. et al., 2000). Hope was assessed using the twelve-item Adult State Hope Scale (Snyder et al., 1996). A series of studies demonstrated internal consistency, high levels of convergent and discriminant validity, and sensitivity (Snyder et al., 1996) and has been used in mental health populations (McGrew, Johannesen, Griss, Born, \& Katuin, 2005; Salyers, Godfrey, et al., 2009).

$\underline{\text { Medical records }}$ were used to extract diagnostic information for consumers. We also extracted appointment data (number of appointments scheduled, missed, and kept) and calculated the number of missed divided by the number scheduled as a measure of disengagement during each period. 
Quantitative Analyses. In order to assure the comparability of the groups (BREATHE vs MI) at baseline concerning demographics and all outcomes for both clinicians and consumers, independent samples t tests for continuous variables and chi-square difference tests for categorical variables were used. To test the effectiveness of the interventions, we used mixed effects modeling to examine BREATHE vs MI intervention effects on the change in clinician burnout and consumer processes, engagement, and outcomes after the intervention, controlling for the baseline score for each outcome and the implementation site. Mixed effects modeling accounts for the nested data structure (consumer or clinician time-dependent outcomes are nested within individuals) (Hesser, 2015). It is superior to traditional methods such as repeated measures ANOVA because it is able to handle nonresponse as well as dropout with full information maximum likelihood estimation (a comparable method to multiple imputation, see Enders, 2010).

In the mixed effects models, we included time and group effects, their interactions, as well as the covariates (i.e., baseline score and implementation site). The time by group interaction effect is the focus of the study because the interaction effect shows whether the two intervention groups differ in terms of the change in the target outcomes over time. In other words, if a significant interaction effect is present, the time effect (improvement in outcome measure over time) is dependent on the group membership (i.e., BREATHE vs MI), and the group that improved more would reflect a more effective method. We completed an intent-totreat analysis. The variables used in the statistical models were carefully examined (e.g., means, standard deviations, outliers, skewness, etc.) to confirm that they met the statistical assumptions of the test statistical models. We also conducted exposure analyses to test whether the levels of BREATHE exposure (e.g., number of sessions attended) impacted the clinician outcomes 
differently over time. In addition, we explored whether the frequency of use and the perceived helpfulness of specific strategies of BREATHE (13 strategies) predicted burnout over time. Finally, we examined whether rates of missed appointments decreased for the consumers who were served by clinicians in the BREATHE condition after the intervention. Analyses were conducted in SAS version 9.3 and tables display means, SD, and beta weights (for time and the interaction of time by group). The p value of less than .05 was applied for significance testing. Our a priori power analysis indicated that 150 clinicians with 450 consumers provide greater than $80 \%$ power to detect a moderate direct effect ( $\beta=.2$; (Muthén \& Muthén, 2002) on clinician burnout and indirect effects on consumer outcomes. The effect size was determined based on the BREATHE pilot study (i.e., Cohen's d $=.43 \&$.65) (Salyers et al., 2011).

Qualitative analyses. Qualitative analyses were used to assess clinician perceptions of the impact of the interventions. We used an emergent content analytic approach (Hsieh \& Shannon, 2005) and included elements of immersion and crystallization (Crabtree \& Miller, 1999). Clinician interviews were transcribed, double checked, and de-identified, removing references to the specific intervention being described. Transcripts were entered into Atlas-ti to help store and facilitate access to data for coding and analysis. Through an iterative, consensusbuilding process, a team reviewed the interview transcripts to identify emergent themes related to the potential impact of the interventions. Initially, we independently read responses to identify possible categories then met several times to discuss our findings and develop a working set of codes. Once we had a defined set of codes, we systematically applied codes to the transcripts and summarized themes.

\section{Results}


We recruited 206 clinicians and 192 were randomized (89 to BREATHE and 103 to MI). As shown in the CONSORT diagram, we had substantial study attrition in the clinician sample, primarily due to clinicians leaving their respective agencies. At the final data collection point (12 months), 61 (69\%) of BREATHE clinicians remained and 66 (64\%) of MI clinicians remained in the study (overall 34\% missing at 12 months). Background characteristics at baseline were examined for the total randomized sample and between clinicians assigned to BREATHE vs MI (See Table 1). The clinician sample was primarily female $(80 \%)$, white $(84 \%)$, and had at least a bachelor's degree (82\%). Most had worked in the mental health field, for their current organization, and in their current position for at least several years (mean of 8.9, 5.0, and 3.3 years, respectively). Clinicians generally worked full time (mean $=41.6$ hours/week). At baseline, clinicians reported low levels of burnout overall (e.g., mean emotional exhaustion of 2.5 corresponds roughly to less than a few times a month). In our samples, the BREATHE group had slightly more males $(\mathrm{n}=25,28 \%)$ compared to the MI group $(\mathrm{n}=14,14 \% ; p=.013)$ and a trend toward less emotional exhaustion at baseline $(p=.068)$, but groups did not differ significantly on any other background variable.

Treatment completion differed significantly between the two groups: BREATHE $=72 \%$ who attended all sessions, versus $\mathrm{MI}=50 \%, \mathrm{X}^{2}=9.97, p=.002$. For BREATHE, 89 were randomized to this condition: $64(72 \%)$ attended all sessions, $10(11 \%)$ attended two of the three sessions, $6(7 \%)$ attended only one session, and $9(10 \%)$ did not attend any sessions. While 103 were randomized to MI, $51(50 \%)$ attended all sessions, $23(22 \%)$ attended two of the three sessions, $16(16 \%)$ attended only one session, and $13(13 \%)$ did not attend any sessions.

Clinician-reported outcomes. In our main analyses comparing clinicians randomized to BREATHE vs MI, we found no advantage for either intervention on burnout (our primary 
outcome) or any of the secondary outcomes (i.e., no interaction effects, See Table 2). Within group changes were noted on a few variables. Contrary to expectations, clinicians in both groups reported trends of marginally increased thoughts about turnover (thoughts of leaving the job in the past 6 months) over time. For both groups, thoughts about turnover increased by .15 units on average every 6 months. The MI group showed significant increases in future intent to leave (likelihood to leave in the next 6 months increased by .11 units every 6 months, $p=.03$ ). In terms of quality of care, the MI group did report increased overall quality of care and increased quality on the subscale assessing patient-centeredness. The levels of treatment exposure (BREATHE or MI sessions attended) did not significantly predict the difference in clinician-reported outcomes over time.

Consumer-reported outcomes. Consumers who were linked to clinicians in BREATHE were compared to consumers who were linked to clinicians in MI. As shown in the CONSORT diagram, 469 consumers were recruited, met eligibility criteria, and were linked with a clinician who was randomized to either BREATHE or MI. The largest reason for lost-to-follow-up for consumers was that research staff were unable to contact them at their last known address or phone number. At the final data collection point (12 months), 168 of 211 consumers (80\%) linked to BREATHE clinicians remained and 210 of 258 consumers (81\%) linked to MI clinicians remained in the study.

Baseline characteristics are shown in Table 3. Overall, consumers averaged 45.2 years of age; approximately half were female, half were white, and most had completed high school (70\%), but few were in paid employment (13\%). Consumers linked to BREATHE clinicians did not differ significantly from those linked with MI clinicians on any background variables. 
As shown in Table 4, we found no patterns suggesting comparative effectiveness for either condition at the consumer level. Two variables did show significant interactions (intervention by time), where the consumers associated with BREATHE clinicians had higher medication adherence than the consumers associated with MI clinicians by 0.20 units $(p=.04)$. However, the consumers associated with MI clinicians had greater increases in patient-centered care by .11 units $(p=.05)$. Both consumer groups improved on a number of variables including patient activation (the increase rate at 6 months was $2.99[p<.001]$ for BREATHE group and 3.10 $[p<.001]$ for MI group), mental health functioning (the increase rate per 6 months was .81 $[p=.08]$ for BREATHE group and $1.01[p=.02]$ ) for MI group, depression (the decrease rate per 6 months was .70 [ $p=.05]$ for BREATHE group and .80 [ $p=.02]$ for MI group), and anxiety (the decrease rate per 6 months was $.79[p=.01]$ for BREATHE group and $.96[p<.001]$ for MI group). Consumers reported increased satisfaction over time (the increase rate per 6 months was $.07[p=.02]$ for BREATHE group and $.08[p<.001]$ for MI group), and consumers associated with MI clinicians reported increased quality of care over time, while consumers associated with clinicians in the BREATHE condition reported slightly decreased quality of care (the decrease rate per 6 months was $.11, p=.05)$. Finally, we found no comparative effectiveness of BREATHE vs MI on the proportion of missed treatment appointments over time.

Qualitative findings from clinician interviews. Three themes were coded to better understand whether the interventions had any perceived impact on burnout, the clinician's personal life, and on interactions with consumers.

In terms of perceived impact on burnout, there were differences between the two groups. The majority of the BREATHE participants interviewed (17 of 21) described reduced burnout in response to the intervention, compared to only 6 of 21 of the MI group. BREATHE participants 
emphasized the intervention helped them to recognize early signs of burnout and to be more intentional in self-care and in clinical work. One quote highlights this well: "it was a way of taking care of myself... and just being kind of more mindful of it and ... of the things that I really need to do to be able to kind of keep everything working and to be productive and to kind of reduce that burnout... knowing that that's part of what we're going to experience, because I think a lot of times people coming into this kind of work, they don't realize that. So actively taking action on it. It really can be really helpful" (respondent \#20). BREATHE also helped them to implement self-care tools more effectively and that reduced subjective feelings of burnout (e.g., "I'm able to kind of stop and think when I get really overwhelmed, some of the specific techniques for the breathing. And like the nostril one [a relaxation breathing exercise] and some things like that" (respondent \#11). Others focused on the feeling that they are not alone or that the training helped remind them to use skills they already know. The four people who did not report reduced burnout in BREATHE emphasized low burnout to begin with "I really hadn't had much burnout prior to the training. I mean there's always stress involved in social work and working with clients. But I don't think that I was experiencing any level of burnout. And that really hasn't changed"' (respondent \#41).

For participants in the MI group, the few who mentioned reduced burnout described an indirect effect, by making consumer interaction easier. For example, a clinician described: "I think it made some of my interactions with my clients easier... this is kind of a new approach to have the conversations that I need to be able to have for my job. But you know, also help them to progress, so I would definitely say it's made my work easier" (respondent \#40). Reasons for the lack of impact were similar to those in BREATHE (e.g., not using the intervention skills, that the skills were not new, or that they were not burned out to begin with). In addition, several 
identified that MI did not directly address burnout or the factors that cause burnout. For example, one participant stated: "And in terms of me feeling, uh, burnout and using some of the skills that we talked about there-I guess I don't necessarily relate the two. Like, we didn't talk a lot about self-care, so I don't think it really had any effect either way” (respondent \#28).

Most participants described positive impacts of the BREATHE $(\mathrm{n}=19)$ and MI $(\mathrm{n}=11)$ interventions on personal life. BREATHE participants described using self-care strategies at home: "I can kind of live in that moment and be in that moment at that time instead of letting outside stressors or work stress kind of invade that personal time" (respondent \#20). For some, perhaps it was more helpful for personal stressors than specifically burnout, as one participant described: "I think personally because when you are stressed, whether it's about work or your personal things, learning how to de-stress is important. So I think for me personally, it helped probably more than professionally" (respondent \#11). For MI participants who reported a positive personal impact, they also described using the skills at home, frequently mentioning the use of MI with family members and friends; for example, participants discussed using value cards to deepen relationships: "Well, when I did it on my husband, I was surprised that what his choices were because they were way different than mine.”(respondent \#15) or to improve communication: "There's some personal relationships that I have that I found the techniques that were discussed and way to approach conversations with them, I found to be helpful" (respondent \#16). For many in the MI group, however, the impact on personal life was minimal: "Not especially. I mean I felt like it was useful for my job to some degree but personally, not so much" (respondent \#22).

For the third area of impact, interaction with consumers, almost all participants (19 in both groups) described positive impacts from their respective training. In the BREATHE group, 
some described using strategies on themselves to be better prepared or attentive for consumers, for example, "Sometimes when I know I'm going to go be with a particularly difficult client, I pause before I go in. I do a little breathing” (respondent \#7). Others described using the skills they learned with the consumers: "I was able to get a lot out of it— able to use a lot of the intervention for myself as well as with the clients that I see" (respondent \#23). Some participants described indirect effects of BREATHE, such as by being less stressed themselves, they would be more effective with the consumers: "I think for me, as my distress levels go up and my burnout levels go up... I'm less connected to them. Am I less empathetic, maybe. So, that's a good thing that anytime I can be less distressed, feeling less burned out, I think I'm going to be better, more helpful for my clients" (respondent \#18). The reasons provided by the MI group were similar, but more direct examples were given in using the specific tools or skills with consumers (which would be expected given the focus of the training), like the value cards, developing discrepancy, or confidence rulers. Some did talk about indirect effects on consumers as well: "And how they respond to you because you're not just going in there and asking a bunch of yes or no questions. It's different. And I think it helps them maybe feel more engaged in like conversation and maybe more open to share sometimes" (respondent \#16).

\section{Discussion}

We set out to test whether providing effective interventions for the health and well-being of clinicians may be another way to improve the quality of care clinicians provide and, ultimately, consumer outcomes. The main hypothesis that the BREATHE intervention would reduce burnout and, therefore, increase patient-centered care processes and outcomes was not supported. Clinician burnout as the primary outcome did not change in response to either intervention. The lack of findings in secondary outcomes, such as consumer care processes and 
outcomes, were understandable because these outcomes were predicated on differences achieved in clinician burnout. The lack of statistically significant change for clinician burnout over time was surprising and disappointing, given prior work with similar populations showing improvements in burnout over time (Salyers et al., 2011). However, in a recently completed RCT, BREATHE did not show comparative effectiveness relative to a person-centered planning workshop, although the BREATHE participants did show significant improvements over time in depersonalization and emotional exhaustion (Rollins et al., 2016). In the context of a strong study design and sufficient power, reasons for the lack of significant findings may still help us address burnout more effectively.

First, it should be noted that, in contrast to the quantitative analyses, the qualitative evaluation suggested that participants in both conditions found the interventions - especially the BREATHE intervention - to have positive benefits for quality of care and for themselves. Interestingly, some clinicians noted in the interviews that positive benefits were even stronger in their personal lives than on the job. BREATHE may give participants new skills (or permission to use existing skills) in the service of self-care. Participants also described increased social connections at work - recognizing they are not alone in experiencing work stress, which can be important for burnout as co-worker support is a predictor of reduced burnout (Crawford, LePine, \& Rich, 2010; Halbesleben, 2006) and related constructs such as reduced satisfaction (Ng \& Sorensen, 2008). In contrast, MI may be more indirect; by providing skills to work more effectively with consumers, some work demands related to consumer care may be reduced. Moreover, participants described that skills are transferrable to other settings (e.g., interacting with family members). Given the stark contrast between interview and survey results, a key question is why the discrepancy? Although it is possible that participants responded more 
positively in interviews than in the online survey due to demand characteristics, we did try to reduce bias by asking neutral questions and having interviewers who were not part of the intervention. We believe that the discrepancy may have more to do with the assessment of burnout in our sample.

Clinicians in this study had a generally low level of burnout even at baseline - much lower than in the original study of BREATHE (Salyers et al., 2011), which showed significant reductions in emotional exhaustion and cynicism. The qualitative interviews also reflect that several people did not recognize themselves as having been "burned out" prior to the training. Thus, while the intervention may have been perceived as helpful, floor effects in the primary outcome measure may have masked any benefit in the quantitative analyses. In line with these findings, burnout was stable over time for both intervention groups. Given that our research design did not include a no-treatment control group, it is possible that the natural course of burnout for clinicians in these agencies involves a worsening of burnout over time. Indeed, turnover rates were fairly high at both agencies $(25 \%$ in one and $40 \%$ in the other during one year). While not all turnover can be attributed to burnout, there are certainly links between them, with burnout being associated with greater likelihood of voluntary turnover (Laschinger \& Fida, 2014; Wright \& Cropanzano, 1998). Moreover, turnover increases the demands of coworkers who stay, such that the low staffing levels and any corresponding burnout for workers left behind can impact consumer outcomes (Cimiotti, Aiken, Sloane, \& Wu, 2012; Vahey, Aiken, Sloane, Clarke, \& Vargas, 2004). Thus, the steady rate of burnout in both conditions over time may represent a positive treatment effect. Future intervention studies could include a no-treatment condition and examine subgroups of participants to better understand the natural course of burnout over time. 
Of course, our lack of findings may also reflect that BREATHE is not effective, at least for this group of participants or for these organizations. The BREATHE program targets individual resilience and draws upon a number of clinical techniques (e.g., mindfulness, deep breathing, cognitive strategies) that many mental health clinicians are already familiar with. Because the initial study (Salyers et al., 2011) was open to all employees, not just clinicians as in the current study, the techniques may have been more impactful for the pilot sample. Alternatively, instead of targeting the individual, effective burnout interventions may need to target organizational changes. One recent review of burnout interventions for medical providers emphasized the utility of both individual and organizational interventions, particularly in combination (West, Dyrbye, Erwin, \& Shanafelt, 2016), while another favored organizational over individual interventions (Panagioti, Panagopoulou, Bower, \& et al., 2017). Many of the organizational interventions described in these reviews included interventions around communication and teamwork, protected time for meaningful work tasks, and improving workload or scheduling; others emphasized task shifting administrative tasks to non-physician team members (Bodenheimer \& Sinsky, 2014). Neither BREATHE nor MI directly address these types of issues.

A key limitation of this study was the low level of clinician burnout at baseline. We conceptualize the BREATHE intervention as one that might reduce or prevent burnout and therefore did not require a minimum burnout level to participate. However, the low initial levels make it difficult to demonstrate significant improvement. We were also limited by a lack of notreatment control. We chose two active comparators, both of which might have a reasonable chance to improve patient-centered care. However, without a no-treatment control, we cannot identify the natural course of burnout in the clinicians in these settings and whether the 
interventions may have prevented a worsening of burnout. The study was also limited by high attrition in the clinician sample, primarily due to staff leaving their agency. Using intent to treat analyses, we analyzed all data available, regardless of whether they received the full interventions. However, the high attrition may have impacted our ability to fully assess the effectiveness.

We are examining supplemental data collected in this study to explore ideas for strengthening the BREATHE intervention (e.g., from the clinician interviews, feedback from training sessions, examining predictors of positive response to BREATHE in this sample and others). However, even if we can strengthen BREATHE, the field is moving toward greater attention to organizational issues as critical to effectively addressing problems with staff wellbeing (Bodenheimer \& Sinsky, 2014; Panagioti et al., 2017; West et al., 2016). Future research should examine both individual and organizational interventions to support clinician well-being. Future studies may also target those in most need of a burnout intervention, for example specifying a minimum level of burnout to participate. However, recruiting the most burned out clinicians poses practical dilemmas as high levels of emotional exhaustion and cynicism would likely interfere with taking on additional training or research tasks.

\section{Conclusions}

In this RCT, BREATHE was not found to be more effective than Motivational Interviewing in reducing burnout or impacting patient-centered care or outcomes. BREATHE was well-received and well-attended (despite high levels of turnover in the study as a whole). Qualitative interviews suggested perceived benefits on clinicians' burnout, personal lives, and how they worked with consumers. However, turnover was problematic, particularly in one site with $40 \%$ turnover of clinicians within one year. Given these high rates of turnover despite the 
provision of BREATHE and MI interventions, organizational level approaches to reducing burnout may be required in order to address both burnout and turnover more comprehensively.

ETHICAL APPROVAL: All procedures performed in studies involving human participants were in accordance with the ethical standards of the institutional and/or national research committee and with the 1964 Helsinki declaration and its later amendments or comparable ethical standards. 


\section{References}

Allsop, S. (2007). What is this thing called motivational interviewing? Addiction, 102(3), 343345. doi:10.1111/j.1360-0443.2006.01712.x

Åström, S., Nilsson, M., Norberg, A., Sandman, P. O., \& Winblad, B. (1991). Staff burnout in dementia care—relations to empathy and attitudes. International Journal of Nursing Studies, 28(1), 65-75. doi:10.1016/0020-7489(91)90051-4

Awa, W. L., Plaumann, M., \& Walter, U. (2010). Burnout prevention: a review of intervention programs. Patient Education and Counseling, 78(2), 184-190. doi:10.1016/j.pec.2009.04.008

Bakker, A. B., \& Demerouti, E. (2007). The job demands-resources model: State of the art. Journal of Managerial Psychology, 22(3), 309-328. doi:10.1108/02683940710733115

Bakker, A. B., Demerouti, E., de Boer, E., \& Schaufeli, W. B. (2003). Job demands and job resources as predictors of absence duration and frequency. Journal of Vocational Behavior, 62(2), 341-356. doi:10.1016/s0001-8791(02)00030-1

Bodenheimer, T., \& Sinsky, C. (2014). From triple to quadruple aim: Care of the patient requires care of the provider. Annals of Family Medicine, 12(6), 573-576. doi:10.1370/afm.1713

Bond, G. R., Witheridge, T. F., Dincin, J., Wasmer, D., Webb, J., \& De Graaf-Kaser, R. (1990). Assertive community treatment for frequent users of psychiatric hospitals in a large city: a controlled study. American Journal of Community Psychology, 18(6), 865-891. doi:10.1007/BF00938068 
Carlson, D. S., Kacmar, K. M., \& Williams, L. J. (2000). Construction and initial validation of a multidimensional measure of work-family conflict. Journal of Vocational Behavior, 56(2), 249-276. doi:10.1006/jvbe.1999.1713

Carson, J., Cavagin, J., Bunclark, J., Maal, S., Gournay, K., Kuipers, E., . . West, M. A. (1999). Effective communication in mental health nurses: Did social support save the psychiatric nurse? Nursing Times Research, 4(1), 31-42. doi:10.1177/136140969900400105

Cimiotti, J. P., Aiken, L. H., Sloane, D. M., \& Wu, E. S. (2012). Nurse staffing, burnout, and health care-associated infection. American Journal of Infection Control, 40(6), 486-490. doi:10.1016/j.ajic.2012.02.029

Crabtree, B. F., \& Miller, W. L. E. (1999). Doing qualitative research: Second edition. Thousand Oaks: Sage Publications.

Crawford, E. R., LePine, J. A., \& Rich, B. L. (2010). Linking job demands and resources to employee engagement and burnout: a theoretical extension and meta-analytic test. Journal of Applied Psychology, 95(5), 834-848. doi:10.1037/a0019364

Diefendorff, J. M., Croyle, M. H., \& Gosserand, R. H. (2005). The dimensionality and antecedents of emotional labor strategies. Journal of Vocational Behavior, 66(2), 339357. doi:http://dx.doi.org/10.1016/j.jvb.2004.02.001

Dreison, K., Luther, L., Bonfils, K. A., McGrew, J. H., Sliter, M. T., \& Salyers, M. P. (2018). Job burnout in mental health providers: A meta-analysis of 35 years of intervention research. Journal of Occupational Health Psychology, 23, 18-30. doi:10.1037/ocp0000047

Druss, B. G., Zhao, L., von Esenwein, S. A., Bona, J. R., Fricks, L., Jenkins-Tucker, S., . . . Lorig, K. (2010). The health and recovery peer (HARP) program: A peer-led intervention 
to improve medical self-management for persons with serious mental illness. Schizophrenia Research, 118(1-3), 264-270. doi:10.1016/j.schres.2010.01.026

Enders, C. K. (2010). Applied missing data analysis. New York, NY: Guilford press.

Fukui, S., Rapp, C. A., Goscha, R., Marty, D., \& Ezell, M. (2014). The perceptions of supervisory support scale. Administration and Policy in Mental Health and Mental Health Services Research, 41(3), 353-359. doi:10.1007/s10488-013-0470-z

Green, C., Perrin, N., Polen, M., Leo, M., Hibbard, J., \& Tusler, M. (2010). Development of the Patient Activation Measure for Mental Health. Administration and Policy in Mental Health and Mental Health Services Research, 37(4), 327-333. doi:10.1007/s10488-0090239-6

Hackman, J. R., \& Oldham, G. R. (1974). The Job Diagnostic Survey: An Instrument for the Diagnosis of Jobs and the Evaluation of Job Redesign Projects. Retrieved from http://search.ebscohost.com/login.aspx?direct=true \&db=eric\&AN=ED099580\&site=ehos $\underline{\text { t-live }}$

Halbesleben, J. R. (2006). Sources of social support and burnout: a meta-analytic test of the conservation of resources model. Journal of Applied Psychology, 91(5), 1134-1145. doi:10.1037/0021-9010.91.5.1134

Halbesleben, J. R., \& Rathert, C. (2008). Linking physician burnout and patient outcomes: exploring the dyadic relationship between physicians and patients. Health Care Management Review, 33(1), 29-39. doi:10.1097/01.HMR.0000304493.87898.72

Heaney, C. A., Price, R. H., \& Rafferty, J. (1995). The caregiver support program: An intervention to increase employee coping resources and enhance mental health. In L. R. 
Murphy, J. J. Hurrell, S. L. Sauter, \& G. P. Keita (Eds.), Job stress interventions (pp. 93108). Washington, DC, US: American Psychological Association.

Hesser, H. (2015). Modeling individual differences in randomized experiments using growth models: recommendations for design, statistical analysis and reporting of results of internet interventions. Internet interventions, 2(2), 110-120.

Hibbard, J. H., Mahoney, E. R., Stockard, J., \& Tusler, M. (2005). Development and testing of a short form of the patient activation measure. Health Services Research, 40(6 Pt 1), 19181930. doi:10.1111/j.1475-6773.2005.00438.x

Hobfoll, S. E. (1989). Conservation of resources: A new attempt at conceptualizing stress. American Psychologist, 44(3), 513-524. doi:10.1037/0003-066X.44.3.513

Hsieh, H. F., \& Shannon, S. E. (2005). Three approaches to qualitative content analysis. Qualitative Health Research, 15(9), 1277-1288. doi:10.1177/1049732305276687

Killian, K. D. (2008). Helping till it hurts? A multimethod study of compassion fatigue, burnout, and self-care in clinicians working with trauma survivors. Traumatology, 14(2), 32-44. doi:10.1177/1534765608319083;

Kroenke, K., Spitzer, R. L., \& Williams, J. B. (2001). The PHQ-9: validity of a brief depression severity measure. Journal of General Internal Medicine, 16(9), 606-613. doi:10.1046/j.1525-1497.2001.016009606.x

Kroenke, K., Spitzer, R. L., Williams, J. B., Monahan, P. O., \& Lowe, B. (2007). Anxiety disorders in primary care: prevalence, impairment, comorbidity, and detection. Annals of Internal Medicine, 146(5), 317-325. doi:10.7326/003-4819-146-5-200703060-00004

Kroenke, K., Theobald, D., Wu, J., Norton, K., Morrison, G., Carpenter, J., \& Tu, W. (2010). Effect of telecare management on pain and depression in patients with cancer: a 
randomized trial. JAMA : The journal of the American Medical Association, 304(2), 163171. doi:10.1001/jama.2010.944

Laschinger, H. K. S., \& Fida, R. (2014). A time-lagged analysis of the effect of authentic leadership on workplace bullying, burnout, and occupational turnover intentions. European Journal of Work and Organizational Psychology, 23(5), 739-753. doi:10.1080/1359432X.2013.804646

Long, C. G., Blackwell, C. C., \& Midgley, M. (1990). An evaluation of two systems of in-patient care in a general hospital psychiatric unit II: measures of staff and patient performance. Journal of Advanced Nursing, 15(12), 1436-1442. doi:10.1111/j.13652648.1990.tb01786.x

Luther, L., Fukui, S., Garabrant, J. M., Rollins, A. L., Morse, G., \& Salyers, M. P. (in press). Measuring quality of care in community mental health: Validation of concordant clinician and client quality of care scales. Journal of Behavioral Health Services \& Research.

Luther, L., Miller, A., Hedrick, H., York, M. M., Firmin, R. L., Morse, G., . . Salyers, M. P. (2016). Client and provider perspectives on the impact of burnout on quality of care and client outcomes in community mental health. Journal of Behavioral and Social Sciences, $3,73-85$.

Maslach, C. (1993). Burnout: A multidimensional perspective. In W. B. Schaufeli, C. Maslach, \& T. Marek (Eds.), Professional burnout: Recent developments in theory and research (pp. 19-32). Washington, DC: Taylor \& Francis.

Maslach, C., Jackson, S. E., \& Leiter, M. P. (1996). Maslach burnout inventory manual. Palo Alto, CA: Consulting Psychologists Press. 
McGrew, J. H., Johannesen, J. K., Griss, M. E., Born, D., \& Katuin, C. (2005). Performancebased funding of supported employment: A multi-site controlled trial. Journal of Vocational Rehabilitation, 23, 81-99.

Miller, W. R., \& Rollnick, S. (2002). Motivational interviewing: Preparing people for change (2nd ed.). New York, NY US: Guilford Press.

Morse, G., Salyers, M., Rollins, A., Monroe-DeVita, M., \& Pfahler, C. (2012). Burnout in mental health services: A review of the problem and its remediation. Administration and Policy in Mental Health and Mental Health Services Research, 39(5), 341-352. doi:10.1007/s10488-011-0352-1

Muthén, L. K., \& Muthén, B. O. (2002). How to use Monte Carlo study to decide on sample size and determine power. Structural Equation Modeling, 9, 599-620. doi:10.1207/S15328007SEM0904_8

Ng, T. W. H., \& Sorensen, K. (2008). Toward a further understanding of the relationships between perceptions of support and work attitudes: a meta analysis. Group \& Organization Management, 33(3), 243-268. doi:10.1177/1059601107313307

Oddie, S., Ousley, L. (2007). Assessing burn-out and occupational stressors in a medium secure service. The British Journal of Forensic Practice, 9(2), 32-48. doi:10.1108/14636646200700011

Panagioti, M., Panagopoulou, E., Bower, P., \& et al. (2017). Controlled interventions to reduce burnout in physicians: A systematic review and meta-analysis. JAMA Internal Medicine, 177(2), 195-205. doi:10.1001/jamainternmed.2016.7674 
Passalacqua, S. A., \& Segrin, C. (2012). The effect of resident physician stress, burnout, and empathy on patient-centered communication during the long-call shift. Health Communication, 27(5), 449-456. doi:10.1080/10410236.2011.606527

Perrone, K. M., Ægisdóttir, S., Webb, L. K., \& Blalock, R. H. (2006). Work-family interface: Commitment, conflict, coping, and satisfaction. Journal of Career Development, 32(3), 286-300. doi:10.1177/0894845305283002

Priebe, S., Fakhoury, W., White, I., Watts, J., Bebbington, P., Billings, J., . . Pan-London Assertive Outreach Study, G. (2004). Characteristics of teams, staff and patients: associations with outcomes of patients in assertive outreach. British Journal of Psychiatry, 185, 306-311. doi:10.1192/bjp.185.4.306

Rohland, B. M. (2000). A Survey of Burnout Among Mental Health Center Directors in a Rural State. Administration and Policy in Mental Health and Mental Health Services Research, 27(4), 221-237. doi:10.1023/a:1021361419155

Rollins, A., Kukla, M., Morse, G., Davis, L., Leiter, M., Monroe-DeVita, M., . . Salyers, M. P. (2016). Comparative effectiveness of a burnout reduction intervention for behavioral health providers. Psychiatric Services, 67(8), 920-923. doi:10.1176/appi.ps.201500220

Ryan, R. M., \& Deci, E. L. (2000). Self-determination theory and the facilitation of intrinsic motivation, social development, and well-being. American Psychologist, 55(1), 68-78. doi:10.1037/0003-066X.55.1.68

Salyers, M. P., Bonfils, K. A., Luther, L., Firmin, R. L., White, D. A., Adams, E. L., \& Rollins, A. L. (2017). The relationship between professional burnout and quality and safety in healthcare: A meta-analysis. Journal of General Internal Medicine, 32(4), 475-482. doi:10.1007/s1 1606-016-3886-9 
Salyers, M. P., Fukui, S., Rollins, A. L., Firmin, R., Gearhart, T., Noll, J. P., . . Davis, C. J. (2015). Burnout and self-reported quality of care in community mental health. Administration and Policy in Mental Health and Mental Health Services Research, 42(1), 61-69. doi:10.1007/s10488-014-0544-6

Salyers, M. P., Godfrey, J. L., McGuire, A. B., Gearhart, T., Rollins, A. L., \& Boyle, C. (2009). Implementing the illness management and recovery program for consumers with severe mental illness. Psychiatric Services, 60(4), 483-490. doi:10.1176/appi.ps.60.4.483

Salyers, M. P., Hudson, C., Morse, G., Rollins, A. L., Monroe-DeVita, M., Wilson, C., \& Freeland, L. (2011). BREATHE: A pilot study of a one-day retreat to reduce burnout among mental health professionals. Psychiatric Services, 62(2), 214-217. doi:10.1176/appi.ps.62.2.214

Salyers, M. P., Matthias, M. S., Spann, C., Lydick, J., Rollins, A. L., \& Frankel, R. M. (2009). The role of patient activation in psychiatric visits. Psychiatric Services, 60(11), 15351539. doi:10.1176/ps.2009.60.11.1535

Salyers, M. P., Rollins, A. L., Kelly, Y.-F., Lysaker, P., \& Williams, J. R. (2013). Job satisfaction and burnout among VA and community mental health workers. Administration and Policy in Mental Health, 40(2), 69-75. doi:10.1007/s10488-011$0375-7$

Siebert, D. C. (2005). Personal and occupational factors in burnout among practicing social workers: Implications for research, practitioners, and managers. Journal of Social Service Research, 32(2), 25-44. doi:10.1300/J079v32n02_02

Smoot, S. L., \& Gonzales, J. J. (1995). Cost-effective communication skills training for state hospital employees. Psychiatric Services, 46(8), 819-822. doi:doi:10.1176/ps.46.8.819; 
Snyder, C. R., Sympson, S. C., Ybasco, F. C., Borders, T. F., Babyak, M. A., \& Higgins, R. L. (1996). Development and validation of the State Hope Scale. Journal of Personality and Social Psychology, 70(2), 321 - 335. doi:10.1037/0022-3514.70.2.321

Sox, H. C., \& Greenfield, S. (2009). Comparative effectiveness research: a report from the Institute of Medicine. Annals of Internal Medicine, 151(3), 203-205. doi:10.7326/00034819-151-3-200908040-00125

Spitzer, R. L., Kroenke, K., Williams, J. B., \& Lowe, B. (2006). A brief measure for assessing generalized anxiety disorder: the GAD-7. Archives of Internal Medicine, 166(10), 10921097. doi:10.1001/archinte.166.10.1092

Spitzer, R. L., Williams, J. B., Kroenke, K., Hornyak, R., \& McMurray, J. (2000). Validity and utility of the PRIME-MD patient health questionnaire in assessment of 3000 obstetricgynecologic patients: the PRIME-MD Patient Health Questionnaire ObstetricsGynecology Study. American Journal of Obstetrics \& Gynecology, 183(3), 759-769. doi:10.1067/mob.2000.106580

Stalker, C., \& Harvey, C. (2002). Professional burnout in social service organizations : a review of theory, research, and prevention. Waterloo, Canada: Social Work, Wilfrid Laurier University.

Thompson, K., Kulkarni, J., \& Sergejew, A. A. (2000). Reliability and validity of a new Medication Adherence Rating Scale (MARS) for the psychoses. Schizophrenia Research, 42(3), 241-247. doi:S0920-9964(99)00130-9 [pii]

Tracey, T. J., \& Kokotovic, A. M. (1989). Factor structure of the Working Alliance Inventory. Psychological Assessment: A Journal of Consulting and Clinical Psychology, 1(3), 207. doi:10.1037/1040-3590.1.3.207 
Vahey, D. C., Aiken, L. H., Sloane, D. M., Clarke, S. P., \& Vargas, D. (2004). Nurse burnout and patient satisfaction. Medical Care, 42(2 Suppl), II57-II66. doi:10.1097/01.mlr.0000109126.50398.5a

Van der Linden, D., Keijsers, G. P. J., Eling, P., \& Van Schaijk, R. (2005). Work stress and attentional difficulties: An initial study on burnout and cognitive failures. Work \& Stress, 19(1), 23-36. doi:10.1080/02678370500065275

Ware, J. E., Jr. , Kosinski, M., \& Keller, S. D. (1996). A 12-item short-form health survey: Construction of scales and preliminary tests of reliability and validity. Medical Care, 34(3), $220-233$. doi:10.2307/3766749

Webster, L., \& Hackett, R. K. (1999). Burnout and leadership in community mental health systems. Administration and Policy in Mental Health and Mental Health Services Research, 26(6), 387-399. doi:10.1023/A:1021382806009

West, C. P., Dyrbye, L. N., Erwin, P. J., \& Shanafelt, T. D. (2016). Interventions to prevent and reduce physician burnout: a systematic review and meta-analysis. The Lancet, 388(10057), 2272-2281. doi:https://doi.org/10.1016/S0140-6736(16)31279-X

Wilensky, G. R. (2006). Developing A Center For Comparative Effectiveness Information. Health Affairs, 25(6), w572-w585. doi:10.1377/hlthaff.25.w572

Williams, G. C., McGregor, H. A., King, D., Nelson, C. C., \& Glasgow, R. E. (2005). Variation in perceived competence, glycemic control, and patient satisfaction: relationship to autonomy support from physicians. Patient Education \& Counseling, 57(1), 39-45. doi:10.1016/j.pec.2004.04.001

Williams, G. C., McGregor, H. A., Sharp, D., Levesque, C., Kouides, R. W., Ryan, R. M., \& Deci, E. L. (2006). Testing a self-determination theory intervention for motivating 
tobacco cessation: supporting autonomy and competence in a clinical trial. Health Psychology, 25(1), 91-101. doi:10.1037/0278-6133.25.1.91

Williams, G. C., McGregor, H. A., Zeldman, A., Freedman, Z. R., \& Deci, E. L. (2004). Testing a self-determination theory process model for promoting glycemic control through diabetes self-management. Health Psychology, 23(1), 58-66. doi:10.1037/02786133.25 .1 .91

Williams, J. W., Jr., Barrett, J., Oxman, T., Frank, E., Katon, W., Sullivan, M., . . Sengupta, A. (2000). Treatment of dysthymia and minor depression in primary care: A randomized controlled trial in older adults. JAMA : The journal of the American Medical Association, 284(12), 1519-1526. doi:10.1001/jama.284.12.1519

Wright, T. A., \& Cropanzano, R. (1998). Emotional exhaustion as a predictor of job performance and voluntary turnover. Journal of Applied Psychology, 83(3), 486-493.

doi:10.1037/0021-9010.83.3.486 